\title{
Nonzero macroscopic magnetization in half-metallic antiferromagnets at finite temperatures
}

\author{
Ersoy Şaşığlu* \\ Institut für Festkörperforschung, Forschungszentrum Jülich, D-52425 Jülich, Germany \\ and Department of Physics, Fatih University, Büyükçekmece, TR-34500 İstanbul, Turkey
}

(Received 4 December 2008; published 25 March 2009)

\begin{abstract}
Combining density-functional theory calculations with many-body Green's-function technique, we reveal that the macroscopic magnetization in half-metallic antiferromagnets does not vanish at finite temperature as for the $T=0$ limit. This anomalous behavior stems from the inequivalent magnetic sublattices which lead to different intrasublattice exchange interactions. As a consequence, the spin fluctuations suppress the magnetic order of the sublattices in a different way leading to a ferrimagnetic state at finite temperatures. Computational results are presented for the half-metallic antiferromagnetic $\mathrm{CrMnZ}(Z=\mathrm{P}, \mathrm{As}, \mathrm{Sb})$ semi-Heusler compounds.
\end{abstract}

DOI: 10.1103/PhysRevB.79.100406

PACS number(s): 75.50.Cc, 71.15.Mb, 75.30.Et, 75.60.-d

Half-metallic antiferromagnets (HM-AFMs) are considered to be the most promising class of materials for spintronics applications. ${ }^{1,2}$ A HM-AFM material is not antiferromagnetic in the usual sense of the term; it is a special case of a ferrimagnet with compensated sublattice magnetization. The existence of the gap in one of the spin channels (either up or down) leads to the complete cancellation of the magnetic moments at zero temperature with a $100 \%$ spin polarization of the charge carriers at the Fermi level. In conventional AFMs the macroscopic spin polarization is zero due to the spin rotational symmetry with the exception of the compounds with broken inversion symmetry such as $\alpha-\mathrm{Fe}_{2} \mathrm{O}_{3}$ in which spin-orbit gives rise to weak ferromagnetism $\left(0.002 \mu_{B}\right)$ due to the canting of the magnetic moments. ${ }^{3,4}$ The HM-AFM materials provide several advantages in device applications with respect to the half-metallic ferromagnets (HM-FMs). For example, they would be perfectly stable spin-polarized electrodes in a junction device. These materials do not give rise to stray fields, and thus no magnetic domain walls are formed. Besides applications in spintronics, HM-AFMs provide a possibility of "single spin superconductivity" due to the spin triplet $(S=1)$ pairing in metallic channel. ${ }^{5}$

The possible existence of HM-AFM was pointed out by van Leuken and de $\mathrm{Groot}^{6}$ in 1995. Based on first-principles calculations authors proposed $\mathrm{CrMnSb}$ and $\mathrm{V}_{7} \mathrm{MnFe}_{8} \mathrm{Sb}_{7} \mathrm{In}$ as candidates for HM-AFMs. Later, Pickett suggested that also the cubic double perovskites $\mathrm{La}_{2} \mathrm{VCuO}_{6}, \mathrm{La}_{2} \mathrm{MnVO}_{6}$, and $\mathrm{La}_{2} \mathrm{MnCoO}_{6}$ are HM-AFMs. ${ }^{7}$ Since then substantial effort has been devoted to find materials with HM antiferromagnetic characteristics. Predicted promising systems include double perovskites, ${ }^{8,9}$ thiospinels, ${ }^{10}$ tetrahedrally coordinated transition-metal-based chalcopyrites, ${ }^{11}$ fullHeusler alloys, ${ }^{12,13}$ and monolayer superlattices. ${ }^{14}$ Not only ordered but also several disordered systems have been shown to be HM-AFMs. ${ }^{15-20}$ In spite of substantial theoretical efforts for designing materials with HM-AFM characteristics and the study of their ground-state electronic and magnetic properties, only few works exist addressing the exchange interactions and magnetic phase transition temperatures in these systems. ${ }^{19,20}$

In this Rapid Communication we investigate the temperature dependence of the magnetization in HM-AFMs employing the many-body Green's-function technique ${ }^{21}$ within Ty- ablikov decoupling scheme. ${ }^{22}$ For computational purposes we consider $\mathrm{CrMn} Z$ ( $Z=\mathrm{P}, \mathrm{As}, \mathrm{Sb})$ semi-Heusler compounds which are the simplest systems among the predicted HMAFMs with two magnetic atoms per unit cell and which are compatible with the existing semiconductors technology. However, present findings are valid for more complicated systems such as double perovskites ${ }^{8,9}$ or diluted antiferromagnetic semiconductors. ${ }^{19,20}$ We show that the macroscopic magnetization in these materials does not vanish at finite temperature in contrast to the zero-temperature limit and conventional AFMs. This peculiar behavior originates from the inequivalent magnetic sublattices in HM-AFMs which lead to different intrasublattice exchange interactions and, as a consequence, spin fluctuations suppress the magnetic order of the sublattices in a different way. Thus, at finite temperature sublattice magnetizations do not compensate each other and all three compounds show ferrimagnetic behavior which seems to be contradictory to our knowledge on finite temperature properties of the magnetic materials. However, these seemingly counterintuitive results can be explained by an analysis of the electronic structure and exchange interactions in these systems.

Ground-state calculations are carried out using the augmented spherical wave method within the generalized gradient approximation to the exchange-correlation potential. Details of the computational scheme can be found in Ref. 23. To provide the basis for further considerations we start with a brief discussion of the electronic structure of the $\mathrm{CrMnZ}$ compounds. Like several Heusler alloys these systems have theoretical equilibrium lattice constants (see Table I) close to the ones of the zinc-blende semiconductors ( $\mathrm{GaP}$ and $\mathrm{GaAs})$.

TABLE I. Lattice parameters and spin magnetic moments (in $\left.\mu_{B}\right)$ for $\mathrm{CrMn} Z(Z=\mathrm{P}, \mathrm{As}, \mathrm{Sb})$. The calculated equilibrium lattice constants are 5.44, 5.71, and $6.08 \AA$ for $Z=P$, As and $S b$, respectively, close to the experimental lattice parameters of GaP, GaAs, and InP.

\begin{tabular}{lccccc}
\hline \hline Compound & $a(\AA)$ & $m_{\mathrm{Cr}}$ & $m_{\mathrm{Mn}}$ & $m_{Z}$ & $m_{\text {Cell }}$ \\
\hline CrMnP & $5.45_{[\mathrm{GaP}]}$ & 1.80 & -1.83 & 0.03 & 0.00 \\
$\mathrm{CrMnAs}$ & $5.65_{[\mathrm{GaAs}]}$ & 2.52 & -2.54 & 0.02 & 0.00 \\
$\mathrm{CrMnSb}$ & $5.87_{[\mathrm{InP}]}$ & 2.74 & -2.76 & 0.02 & 0.00 \\
\hline \hline
\end{tabular}


Thus, calculational results will be presented for the latter case since these semiconductors might be considered as possible substrates to grow these materials. ${ }^{24}$ All compounds under study have 18 valance electrons per unit cell and calculations show that the total spin moments given in Table I are exactly zero in agreement with the Slater-Pauling behavior for ideal half metals. ${ }^{25}$ Simultaneously, the spin magnetic moments of $\mathrm{Cr}$ and $\mathrm{Mn}$ atoms are antiparallel and these compounds are ferrimagnets with compensated sublattice magnetizations. The origin of the HM gap in Heusler alloys has been well understood and the reader is referred to Ref. 25 since the same discussion is valid for the present systems.

To study interatomic exchange interactions we map the complex itinerant electron problem onto a classical Heisenberg Hamiltonian $H=-\Sigma_{\mu, \nu} \Sigma_{\mathbf{R}, \mathbf{R}^{\prime}} J_{\mathbf{R R}^{\prime}}^{\mu \nu} s_{\mathbf{R}^{\mu}}^{\mu} s_{\mathbf{R}^{\prime}}^{\nu}$, where $\mu \mathbf{R}$ $\neq \nu \mathbf{R}^{\prime}$ and the indices $\mu$ and $\nu$ represent different sublattices. $\mathbf{R}$ and $\mathbf{R}^{\prime}$ are the lattice vectors specifying the atoms within the sublattices, and $s_{\mathbf{R}}^{\mu}$ is the unit vector pointing in the direction of the magnetic moment at site $(\mu, \mathbf{R})$. Heisenberg exchange parameters $J_{\mathbf{R R}^{\prime}}^{\mu \nu}$ are calculated employing the frozen-magnon technique as described in Ref. 23. Extensive investigations on the multi-sublattice Heusler alloys have shown that there are several exchange interactions which coexist and which are mixed together. ${ }^{23,26-28}$ To simplify the discussion let us write the total magnetic exchange field acting on the sublattice $\mu$ as $J_{\text {total }}^{\mu} \sim J_{\text {direct }}^{(\mu \nu)}+J_{\text {indirect }}^{(\mu \nu)}+J_{\text {indirect }}^{(\mu \mu)}$, where the first two terms represent the direct and indirect exchange couplings between different sublattices. The former (direct coupling) provides the leading contribution to the total exchange coupling and determines the character of the magnetic state, while the latter contributes little and is not so important. On the other hand, the last term, i.e., the intrasublattice coupling is of particular importance because it is responsible for the appearance of a net macroscopic magnetization at finite temperature in HM-AFMs. It should be noted here that in reality the situation is not so simple, the exchange field acting on the sublattices should be determined from the solution of a matrix equation.

Let us start with the discussion of the last term. Because of the large distance between $\mathrm{Cr}-\mathrm{Cr}(\mathrm{Mn}-\mathrm{Mn})$ atoms this coupling is indirect mediated by the conduction electrons. In Fig. 1 we present the calculated $\mathrm{Cr}-\mathrm{Cr}$ and $\mathrm{Mn}-\mathrm{Mn}$ Heisenberg exchange parameters as a function of the distance. As seen, due to inequivalent magnetic sublattices the pattern of $\mathrm{Cr}-\mathrm{Cr}$ and $\mathrm{Mn}-\mathrm{Mn}$ exchange parameters shows very different behavior. Although the former has ferromagnetic character, the latter shows oscillatory behavior so that the ferromagnetic and antiferromagnetic contributions partly compensate each other, giving rise to a small contribution into the total exchange field acting on the Mn sublattice. In Table II we present the intrasublattice $\left(J_{0}^{\mu}=\Sigma_{\mathbf{R} \neq 0} J_{0 \mathbf{R}}^{\mu \mu}\right)$ as well as the intersublattice $\left(J_{0}^{\mu \nu}=\Sigma_{\mathbf{R}} J_{0 \mathbf{R}}^{\mu \nu}\right)$ on-site exchange coupling parameters. The on-site $\mathrm{Cr}-\mathrm{Cr}$ and $\mathrm{Mn}-\mathrm{Mn}$ exchange couplings are rather different, and this difference will be reflected as a net macroscopic magnetization at finite temperatures (see Fig. 2 ). It is worth to note that in conventional AFMs this coupling is the same for both sublattices. The increase in the strength of the exchange interactions and correspondingly of the critical temperatures in the P-As-Sb sequence can be ex-

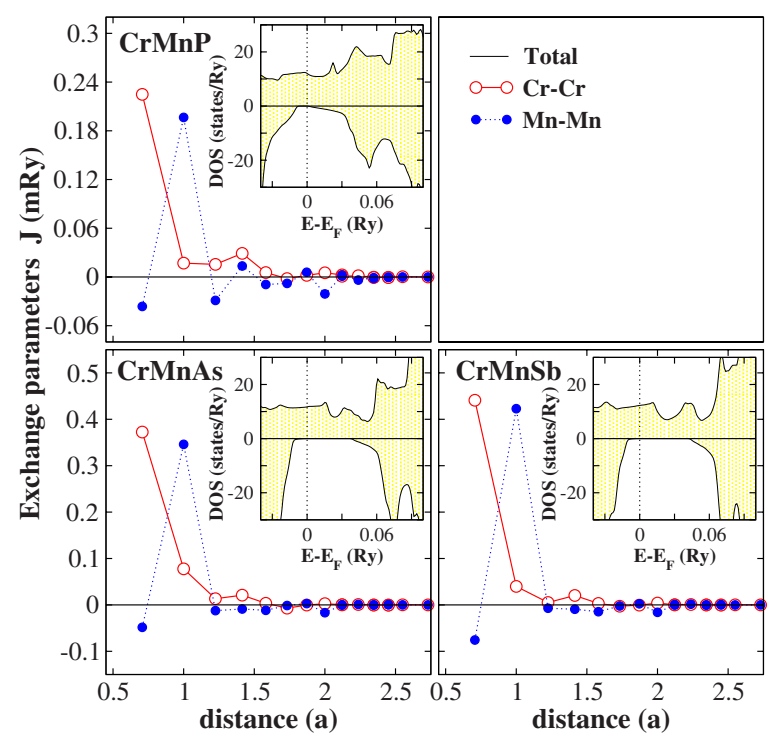

FIG. 1. (Color online) Intrasublattice $\mathrm{Cr}-\mathrm{Cr}$ and $\mathrm{Mn}-\mathrm{Mn}$ exchange interactions in $\mathrm{CrMn} Z(Z=\mathrm{P}, \mathrm{As}, \mathrm{Sb})$ as a function of distance. In the insets we show spin-resolved total density of states around Fermi level.

plained by the increase in the magnetic moments (see Table I). Moreover, due to the presence of the HM gap the exchange interactions quickly decay. ${ }^{26,27}$

In contrast to the intrasublattice exchange interactions, the intersublattice $(\mathrm{Cr}-\mathrm{Mn})$ ones behave very differently. Due to the smaller $\mathrm{Cr}-\mathrm{Mn}$ distance a very strong antiferromagnetic nearest-neighbor direct interaction takes place between these atoms which is about 1 order of magnitude larger than the nearest-neighbor $\mathrm{Cr}-\mathrm{Cr}$ coupling and is responsible for the formation of the ferrimagnetic state. The interactions between further nearest neighbors are very small. The on-site Cr-Mn exchange couplings presented in Table II are about 4 and 20 times larger than the $\mathrm{Cr}-\mathrm{Cr}$ and $\mathrm{Mn}-\mathrm{Mn}$ ones, respectively. Note that the ferromagnetic intrasublattice exchange interactions further stabilize the ferrimagnetic order. The antiferromagnetic coupling of the $\mathrm{Cr}$ and $\mathrm{Mn}$ atoms can be qualitatively explained on the basis of the following facts: First, half-filled shells tend to yield a strong trend toward antiferromagnetism, and second, exchange coupling in $3 d$ transition metals obeys the semiphenomenological BetheSlater-Néel curve which predicts antiferromagnetism in the case of small interatomic distances. ${ }^{29}$ Indeed, the $\mathrm{Cr}-\mathrm{Mn}$ distance $\left(d_{[\mathrm{Cr}-\mathrm{Mn}]}=2.36-2.54 \AA\right)$ in the present systems is comparable with the $\mathrm{Cr}-\mathrm{Cr}$ distance $\left(d_{[\mathrm{Cr}-\mathrm{Cr}]}=2.52 \AA\right)$ in the an-

TABLE II. On-site exchange parameters (in mRy) and estimated critical temperatures (in K) within RPA for quantum $\left(T_{C}^{[Q]}\right.$ ) and classical $\left(T_{C}^{[C]}\right)$ spins for $\operatorname{CrMn} Z(Z=\mathrm{P}, \mathrm{As}, \mathrm{Sb})$.

\begin{tabular}{lccccc}
\hline \hline Compound & $J_{0}^{[\mathrm{Cr}-\mathrm{Cr}]}$ & $J_{0}^{[\mathrm{Mn}-\mathrm{Mn}]}$ & $J_{0}^{[\mathrm{Cr}-\mathrm{Mn}]}$ & $\begin{array}{c}T_{C}^{[Q]} \\
(\mathrm{K})\end{array}$ & $\begin{array}{c}T_{C}^{[C]} \\
(\mathrm{K})\end{array}$ \\
\hline $\mathrm{CrMnP}$ & 3.80 & 0.03 & -12.99 & 2530 & 1264 \\
$\mathrm{CrMnAs}$ & 5.55 & 0.84 & -19.50 & 2610 & 1566 \\
$\mathrm{CrMnSb}$ & 5.96 & 1.06 & -20.16 & 2986 & 1792 \\
\hline \hline
\end{tabular}




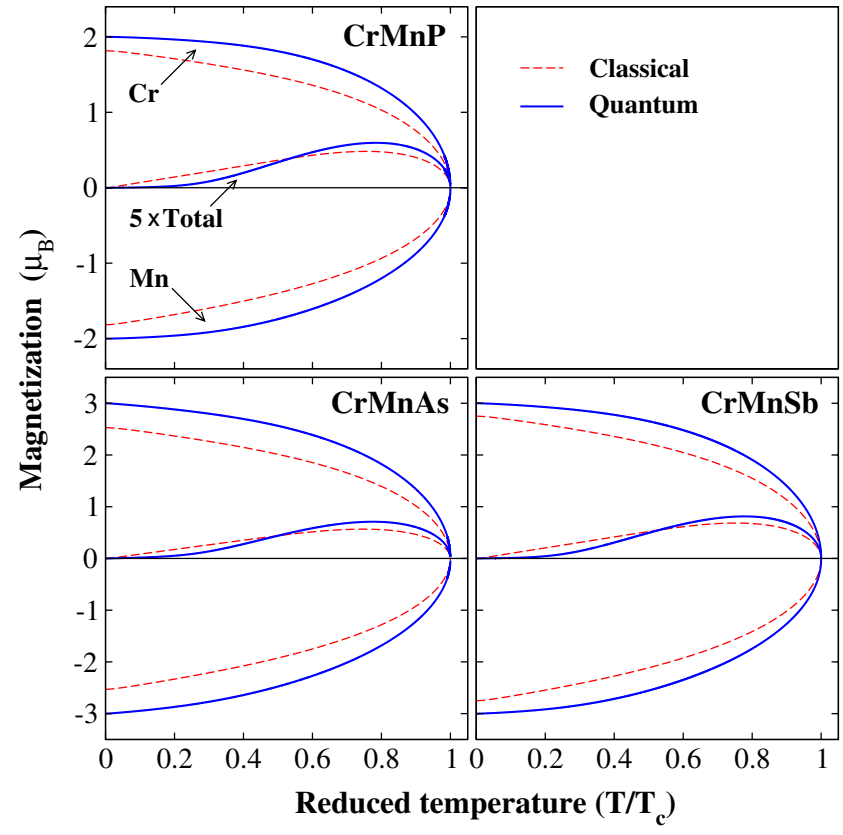

FIG. 2. (Color online) The calculated temperature dependence of the sublattice and total magnetization for $\operatorname{CrMnZ}(Z=\mathrm{P}, \mathrm{As}, \mathrm{Sb})$. The calculations are performed for both classical and quantum Hamiltonians. The temperature is given in reduced form and the total magnetization is scaled up by a factor of 5 .

tiferromagnetic bcc $\mathrm{Cr}$ and both magnetic atoms posses halffilled $3 d$ shells, thus, antiferromagnetic Cr-Mn coupling is expected.

With calculated exchange parameters in hand, now we can study temperature dependence of the magnetization employing the methods of statistical mechanics to the Heisenberg Hamiltonian. We use the many-body Green's-function technique $^{21}$ within Tyablikov decoupling scheme ${ }^{22}$ [also known as the random-phase approximation (RPA)] as described in Ref. 28. Note that RPA takes into account only transverse spin fluctuations and the spin-flip Stoner excitations (longitudinal spin fluctuations) are neglected. However, available experimental data on Heusler alloys have shown that these latter excitations are well separated in energy from the former one (spin waves) due to large exchange splitting $\Delta(\Delta \sim 2-3 \mathrm{eV}) .{ }^{30}$ In addition to this the presence of $\mathrm{HM}$ gap prevents spin-flip transitions. Thus, Stoner excitations do not play an important role in thermodynamics of the present systems and the RPA method is well grounded. We consider both classical-spin and quantum-spin cases. In the classicalspin calculations the obtained values of the magnetic moments (see Table I) are used, while for the quantummechanical case we assign integer values to the atomic moments: $2 \mu_{B}(S=1)$ (for $\mathrm{Cr}$ and $\mathrm{Mn}$ ) in $\mathrm{CrMnP}$ and $3 \mu_{B}$ $(S=3 / 2)$ in CrMnAs and CrMnSb. In quantum-spin case the thermal average of the sublattice magnetization is given by the Callen's expression ${ }^{31}$

$$
\left\langle\hat{s}_{\mu}^{z}\right\rangle=\frac{\left(S_{\mu}-\Phi_{\mu}\right)\left(1+\Phi_{\mu}\right)^{2 S_{\mu}+1}+\left(S_{\mu}+1+\Phi_{\mu}\right) \Phi_{\mu}^{2 S_{\mu}+1}}{\left(1+\Phi_{\mu}\right)^{2 S_{\mu}+1}-\left(\Phi_{\mu}\right)^{2 S_{\mu}+1}},
$$

where $\Phi_{\mu}$ is an auxiliary function. As the $\Phi_{\mu}$ depends on the $\left\langle\hat{s}_{\mu}^{z}\right\rangle$ as well as $\left\langle\hat{s}_{\nu}^{z}\right\rangle$ to be determined, the above equation forms a self-consistency problem to be solved by iteration. Note that for classical spins the Callen's expression is reduced to the Langevin function. ${ }^{28}$ In Fig. 2 we present the calculated temperature dependence of the sublattice and total magnetization for $\mathrm{CrMn} Z(Z=\mathrm{P}, \mathrm{As}, \mathrm{Sb})$. The temperature is given in reduced form and the total magnetization is scaled up by a factor of 5 . As seen, the spin fluctuations suppress the magnetic order of the sublattices differently, i.e., the magnetization of the Mn sublattice decreases faster than the $\mathrm{Cr}$ one and as a result the total magnetization does not vanish at finite temperature in contrast to the $T=0$ limit. This behavior can be traced back to the different exchange fields acting on the sublattices (see Table II). The total magnetization shows nonmonotonous behavior, i.e., first, it increases with increasing temperature up to $0.8 T_{C}$ and then decreases much faster and finally becomes zero at the critical point. For quantum-mechanical case the calculated total magnetic moment around $0.8 T_{C}$ is $0.1 \mu_{B}-0.16 \mu_{B} \quad\left(0.006 \mu_{B}-0.01 \mu_{B}\right.$ around room temperature) which is about 2 orders of magnitude larger than the spin-orbit-coupling-induced weak magnetic moment $\left(0.002 \mu_{B}\right)$ in $\alpha-\mathrm{Fe}_{2} \mathrm{O}_{3} \cdot{ }^{3}$ We should also note that in these systems not only the ideal case of zero macroscopic magnetization but also half metallicity is limited to the $T=0.2,32,33$

The nature of the spin (quantum or classical) plays an important role in the temperature behavior of the magnetization curves. In the quantum case, the magnetization drops slower than the classical case, and thus, the calculated $T_{C}$ values are larger by a factor of $(S+1) / S$ entering the RPA expression (see Ref. 28). Note that this factor becomes unity for classical spins $[(S+1) / S \rightarrow 1$ for $S \rightarrow \infty]$. Another important point which is outside the scope of the present work is that in both treatments we use the exchange parameters estimated within the picture of classical atomic moments. However, it is possible that the values of the exchange parameters must be modified for the use in the quantum-mechanical calculations. In general, the classical calculation provides reasonable values of the critical temperature compared with experiment, while the quantum-mechanical treatment gives better form of the temperature dependence of the magnetization. ${ }^{28}$ The calculated critical temperatures within RPA are presented in Table II. We notice that the predicted $T_{C}$ values of the CrMnAs and $\mathrm{CrMnSb}$ are even higher than the fcc Co which possesses the highest critical temperature (1400 K) among all known magnetic materials. This is not surprising because available experimental and theoretical data have shown that the critical temperatures of HM ferromagnets (or ferrimagnets) scales linearly with the average value of the magnetic moment per atom in the unit cell. ${ }^{34}$ In this respect $\mathrm{Co}_{2} \mathrm{FeSi}$ possesses the largest average magnetic moment per atom of $1.5 \mu_{B}$ with an experimental $T_{C}$ of 1100 $\mathrm{K}$. However, in CrMnAs ( $\mathrm{CrMnSb})$ the average value of the absolute magnetic moment per atom is $1.68 \mu_{B}\left(1.83 \mu_{B}\right)$ which is larger than the corresponding value in $\mathrm{Co}_{2} \mathrm{FeSi}$, and thus such high critical temperatures are expected. Finally we should note that so far discussion is based on the assumption that $\mathrm{CrMnZ}$ compounds possess $C 1_{b}$-type ordered crystal structure and the effect of disorder is completely ignored. However, in reality disorder exists in various forms such as defects, antisites, or atomic swaps which reduce not only the 
spin polarization at the Fermi level but also the magnetic phase transition temperature. ${ }^{35}$ For example, migration of the $\mathrm{Cr}$ atoms to the vacant sublattice is expected to reduce $T_{C}$ substantially since as shown in Ref. 13 the $L 2_{1}$-type $\mathrm{Cr}_{2} \mathrm{MnZ}$ compounds have $T_{C}$ values around room temperature.

In summary, we have studied the temperature dependence of the magnetization in HM-AFM CrMnZ $(Z=\mathrm{P}, \mathrm{As}, \mathrm{Sb})$ compounds employing the many-body Green's-function technique. We have shown that the macroscopic magnetization in these systems does not vanish at finite temperature in contrast to the $T=0$ limit. This anomalous behavior stems from the inequivalent magnetic sublattices in HM-AFMs which lead to different intrasublattice exchange interactions, and as a result, spin fluctuations suppress the magnetic order of the sublattices in a different way. Thus, at finite temperatures, the sublattice magnetizations do not compensate each other and all three compounds show ferrimagnetic behavior. Moreover, the combination of large HM gaps, high $T_{C}$ values and very small macroscopic magnetization around room temperature makes $\mathrm{CrMnAs}$ and $\mathrm{CrMnSb}$ promising candidates for spintronics applications.

Fruitful discussions with L. Sandratskii, I. Galanakis, and Ph. Mavropoulos are greatly acknowledged. *e.sasioglu@fz-juelich.de

${ }^{1}$ W. E. Pickett and J. S. Moodera, Phys. Today 54(5), 39 (2001).

${ }^{2}$ M. I. Katsnelson, V. Yu. Irkhin, L. Chioncel, A. I. Lichtenstein, and R. A. de Groot, Rev. Mod. Phys. 80, 315 (2008).

${ }^{3}$ L. M. Sandratskii and J. Kübler, Europhys. Lett. 33, 447 (1996).

${ }^{4}$ V. V. Mazurenko and V. I. Anisimov, Phys. Rev. B 71, 184434 (2005).

${ }^{5}$ W. E. Pickett, Phys. Rev. Lett. 77, 3185 (1996).

${ }^{6}$ H. van Leuken and R. A. de Groot, Phys. Rev. Lett. 74, 1171 (1995).

${ }^{7}$ W. E. Pickett, Phys. Rev. B 57, 10613 (1998).

${ }^{8}$ J. H. Park, S. K. Kwon, and B. I. Min, Phys. Rev. B 65, 174401 (2002).

${ }^{9}$ Y. K. Wang and G. Y. Guo, Phys. Rev. B 73, 064424 (2006).

${ }^{10}$ M. S. Park, S. K. Kwon, and B. I. Min, Phys. Rev. B 64, 100403(R) (2001).

${ }^{11}$ M. Nakao, Phys. Rev. B 74, 172404 (2006).

${ }^{12}$ S. Wurmehl, H. C. Kandpal, G. H. Fecher, and C. Felser, J. Phys.: Condens. Matter 18, 6171 (2006).

${ }^{13}$ I. Galanakis, K. Özdoğan, E. Şaşıŏlu, and B. Aktaş, Phys. Rev. B 75, 172405 (2007).

${ }^{14}$ M. Nakao, Phys. Rev. B 77, 134414 (2008).

${ }^{15}$ I. Galanakis, K. Özdoğan, E. Şaşığlu, and B. Aktaş, Phys. Rev. B 75, 092407 (2007).

${ }^{16}$ D. Ködderitzsch, W. Hergert, Z. Szotek, and W. M. Temmerman, Phys. Rev. B 68, 125114 (2003).

${ }^{17}$ Y.-m. Nie and X. Hu, Phys. Rev. Lett. 100, 117203 (2008).

${ }^{18}$ H. Akai and M. Ogura, Phys. Rev. Lett. 97, 026401 (2006).

${ }^{19}$ L. Bergqvist and P. H. Dederichs, J. Phys.: Condens. Matter 19, 216220 (2007).

${ }^{20}$ M. Ogura, C. Takahashi, and H. Akai, J. Phys.: Condens. Matter 19, 365226 (2007).

${ }^{21}$ P. Fröbrich and P. J. Kuntz, Phys. Rep. 432, 223 (2006).

${ }^{22} \mathrm{~S}$. V. Tyablikov, Methods of Quantum Theory of Magnetism (Ple- num Press, New York, 1967).

${ }^{23}$ E. Şaşığlu, L. M. Sandratskii, and P. Bruno, Phys. Rev. B 70, 024427 (2004).

${ }^{24}$ Among the considered compounds only CrMnSb is experimentally synthesized with a crystal structure different than the $C 1_{b}$ type semi-Heusler one [J. H. Wijngaard, C. Haas, and R. A. de Groot, Phys. Rev. B 45, 5395 (1992)]. However, state-of-the-art experimental methods for the synthesis of materials such as the molecular-beam epitaxy make possible the growth of these compounds in metastable phases as thin films or multilayers where the substrate determines the lattice parameter.

${ }^{25} \mathrm{I}$. Galanakis and Ph. Mavropoulos, J. Phys.: Condens. Matter 19, 315213 (2007).

${ }^{26}$ J. Rusz, L. Bergqvist, J. Kudrnovský, and I. Turek, Phys. Rev. B 73, 214412 (2006).

${ }^{27}$ J. Kudrnovský, V. Drchal, I. Turek, and P. Weinberger, Phys. Rev. B 78, 054441 (2008).

${ }^{28}$ E. Şaşığlu, L. M. Sandratskii, P. Bruno, and I. Galanakis, Phys. Rev. B 72, 184415 (2005).

${ }^{29}$ R. Skomski, Simple Models of Magnetism (Oxford University Press, New York, 2008).

${ }^{30}$ P. J. Webster and K. R. A. Ziebeck, in Alloys and Compounds of d-Elements with Main Group Elements, Part 2, LandoltBörnstein, New Series, Group III, Vol. 19, edited by H. R. J. Wijn (Springer, Berlin, 1988), part C.

${ }^{31}$ H. B. Callen, Phys. Rev. 130, 890 (1963).

${ }^{32}$ P. A. Dowben and R. Skomski, J. Appl. Phys. 93, 7948 (2003).

${ }^{33}$ M. Ležaić, P. Mavropoulos, J. Enkovaara, G. Bihlmayer, and S. Blugel, Phys. Rev. Lett. 97, 026404 (2006).

${ }^{34}$ J. Kübler, G. H. Fecher, and C. Felser, Phys. Rev. B 76, 024414 (2007).

${ }^{35}$ B. Alling, S. Shallcross, and I. A. Abrikosov, Phys. Rev. B 73, 064418 (2006). 